\title{
Eosinophilic myocarditis presenting with hypoactive delirium and cardioembolic stroke
}

\author{
Ka Hong Chan MD, Paul Gibson MD
}

Cite as: CMAJ 2019 October 21;191:E1159-63. doi: 10.1503/cmaj.190669

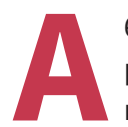

64-year-old woman with normal baseline functioning presented to hospital with an altered level of consciousness. She had a history of hypertension (perindopril $8 \mathrm{mg} / \mathrm{d}$ ), gout (allopurinol $200 \mathrm{mg} / \mathrm{d}$ ) and anxiety (sertraline $100 \mathrm{mg} / \mathrm{d}$ ). She was brought to the hospital by her husband because of a 3-day history of being in a hypoactive state with urinary and stool incontinence. Her husband reported no preceding infectious, allergic or constitutional symptoms. Family history and rheumatologic review of systems were noncontributory, and the patient had no recent history of rashes. There had been no out-of-country travel in the preceding 2 years.

On admission, the patient's temperature was $36.3^{\circ} \mathrm{C}$, she had a regular heart rate of 103 beats $/ \mathrm{min}$, her blood pressure was $128 / 78 \mathrm{~mm} \mathrm{Hg}$ and oxygen saturation was $95 \%$ on room air. Her glucose level was 5.7 (normal 3.3-11.0) mmol/L. The patient's score on the Glasgow Coma Scale was 15/15, but she was inattentive and oriented to only her name. A neurologic examination was limited owing to lack of cooperation. The patient's neck was supple. Pupils were equal and reactive, with no gaze preference or nystagmus. She was unable to squeeze her left hand, and had hypertonia of the left lower extremity and a unilateral upgoing Babinski sign. On the right side, she had antigravity strength. She was hyperreflexic (graded at 3/4) throughout. There were no features of heart failure. The examination was otherwise unremarkable.

Initial laboratory investigations showed a normal complete blood count except for an elevated leukocyte count of $15.7 \times 10^{9} / \mathrm{L}$ owing to an eosinophil count of $6.3($ normal $<0.7) \times 10^{9} / \mathrm{L}$. The patient's complete blood count had been normal 4 months earlier. Electrolytes, creatinine, lipase, liver enzymes and vitamin $B_{12}$ levels were within the normal ranges, and thyroid-stimulating hormone was minimally elevated at 4.07 (normal 0.20-4.00) mIU/L. Importantly, her C-reactive protein (CRP) level was substantially elevated at 110.3 (normal < 8) $\mathrm{mg} / \mathrm{L}$, and serial troponin levels ranged from 1620 to 1780 (normal < 14) ng/L without any clear trend. Electrocardiography showed sinus tachycardia with nonspecific ST and T wave changes in the inferolateral leads. Chest radiography and computed tomography angiography of the head and neck were both normal. Magnetic resonance imaging (MRI), however, showed multiple foci of restricted diffusion within the cerebral hemispheres, basal ganglia and posterior fossa, suggestive of cardioembolic phenomena (Figure 1).

The patient was initially given heparin for possible cardioembolic stroke, and ceftriaxone, vancomycin and acyclovir for

\section{KEY POINTS}

- Hypereosinophilic syndrome is a rare condition manifested by hypereosinophilia with ensuing end-organ damage secondary to tissue infiltration and inflammation by these cells; cardiac involvement from hypereosinophilic syndrome is known as eosinophilic myocarditis.

- The pathophysiology of eosinophilic myocarditis is characterized by 3 phases: acute necrosis (asymptomatic), thrombosis (presents with secondary embolic phenomenon) and fibrosis (most common presentation; presents with cardiomyopathy).

- Treatment of eosinophilic myocarditis relies on elucidating the underlying cause of eosinophilia, which can be broadly categorized as idiopathic, hypersensitivity, rheumatologic, neoplastic and infectious causes.

- The cornerstone of eosinophilic myocarditis management involves corticosteroids with or without anticoagulation, although evidence remains sparse.

potential infectious endocarditis or meningoencephalitis. An extensive infectious workup was undertaken. Urinalysis was clear. Blood, stool (including ova and parasites) and urine cultures were negative. Cerebrospinal fluid (CSF) analysis showed a leukocyte count of $1 \times 10^{6} / \mathrm{L}$ and a CSF protein level of 0.47 (normal $0.15-0.45) \mathrm{g} / \mathrm{L}$ but was negative for culture and viral testing. HIV testing was not performed owing to lack of risk factors and ability to consent. Transthoracic echocardiography did not show any left ventricular thrombus, effusions, valvular abnormalities or abnormalities in systolic function. Telemetry for more than 96 hours showed no signs of arrhythmia. Electroencephalography showed only generalized slowing.

Despite discontinuation of all her home medications, the patient's eosinophilia persisted for 7 days. Immunologic investigations included negative testing for antinuclear antibodies, extractable nuclear antigens, rheumatoid factor and antineutrophil cytoplasmic antibodies. Serum immunoglobulin E levels were normal at 19.9 (normal < 160.0) klU/L, and peripheral blood flow cytometry showed no evidence of a B-cell neoplasm. Computed tomography of the patient's chest, abdomen and pelvis did not show any lymphadenopathy or occult malignancy. Cytogenetic investigations for platelet-derived growth factor receptor $\alpha$ and Janus kinase 2 mutations were also negative. 
As there was no clear diagnosis despite an exhaustive workup, the patient ultimately underwent an endomyocardial

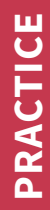
biopsy. Cardiac MRI could not be conducted because the patient was unable to follow commands for a valid study. The biopsy showed eosinophilic infiltration of the endomyocardium, with associated myocyte damage and mural thrombi formation, consistent with a diagnosis of eosinophilic myocarditis (Figure 2). The patient was subsequently started on

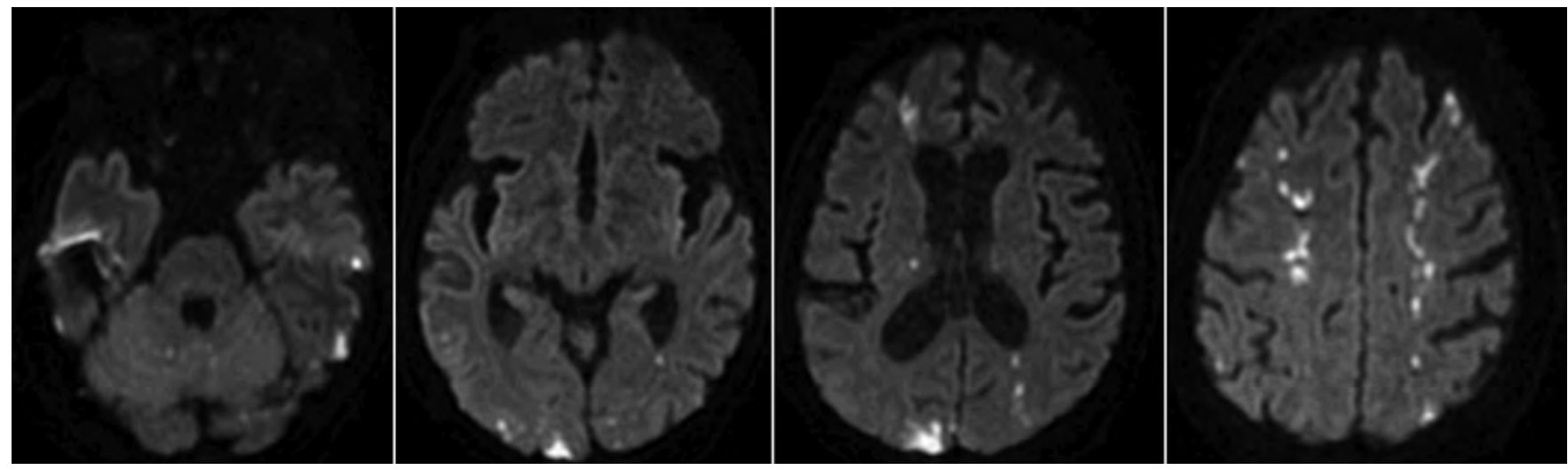

Figure 1: Diffusion-weighted magnetic resonance imaging in a 64-year-old woman with altered level of consciousness showing multiple foci of restricted diffusion in the cerebral hemispheres (frontal, parietal, occipital and temporal), basal ganglia and posterior fossa, suggestive of a multiterritorial stroke.

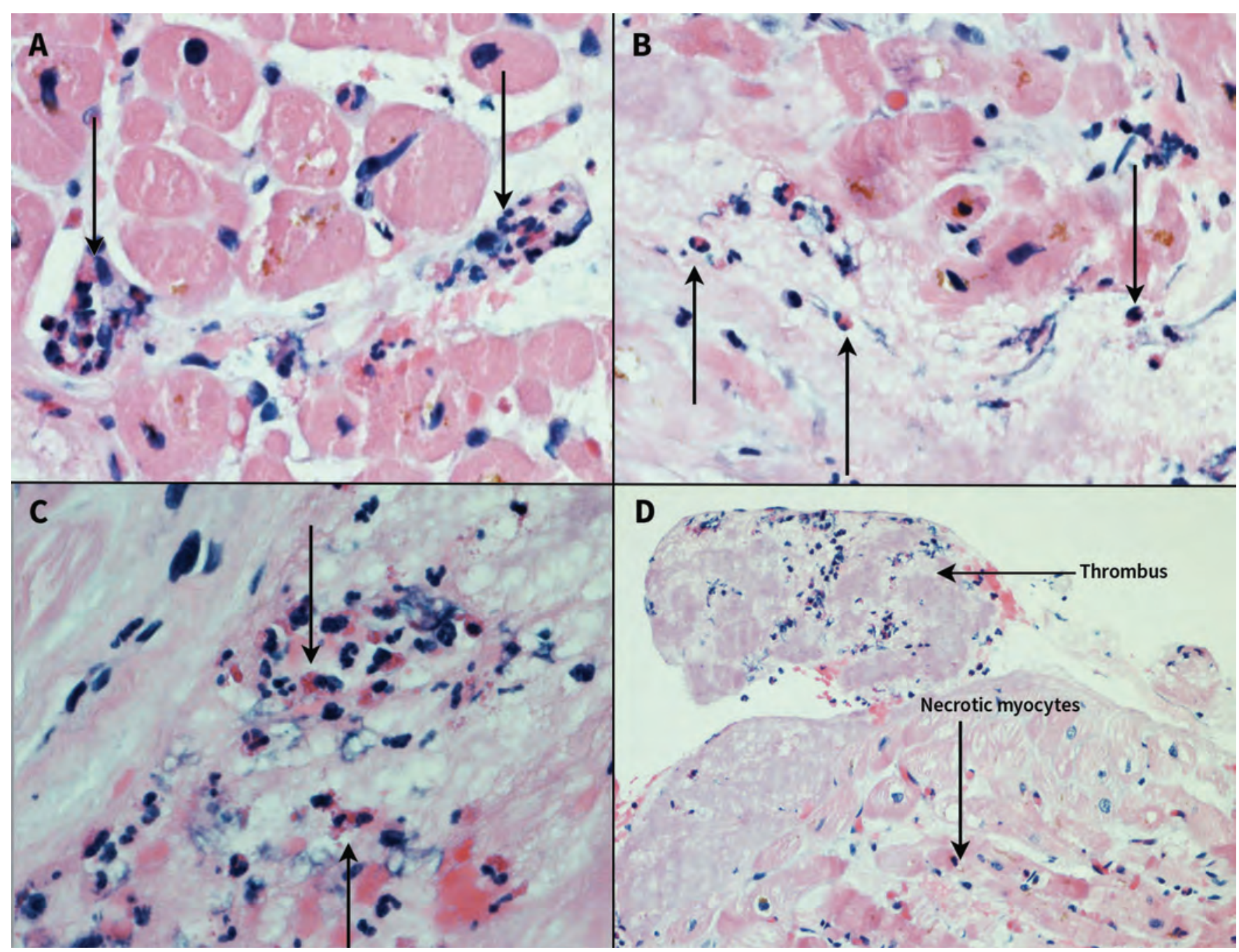

Figure 2: Pathologic findings of eosinophilic myocarditis showing A) intravascular eosinophils migrating into the myocardium (hematoxylin and eosin stain, original magnification $\times 400$ ), B) presence of eosinophils in the myocardium (hematoxylin and eosin stain, original magnification $\times 400$ ), C) eosinophils with degranulation within the myocardium (hematoxylin and eosin stain, original magnification $\times 400$ ) and D) development of mural thrombus within the endocardium (hematoxylin and eosin stain, original magnification $\times 200$ ). 
methylprednisolone $1 \mathrm{~g}$ intravenously for 3 days followed by a prednisone taper starting at $1 \mathrm{mg} / \mathrm{kg}$. By the following day, her eosinophil count completely normalized to $0.1 \times 10^{9} / \mathrm{L}$. Over the course of a month, the patient's CRP level returned to normal and her troponin level decreased to $50 \mathrm{ng} / \mathrm{L}$. Clinically, her level of consciousness slowly improved, and she was eventually able to participate in simple conversations. Unfortunately, the patient remained weak owing to the extent of her multifocal stroke, and the resulting persistent cognitive dysfunction severely limited her rehabilitation. For that reason, a bone marrow biopsy was never performed, as it was felt that management would not likely be altered. The patient was discharged to a long-term care facility.

\section{Discussion}

Eosinophils are a subtype of myeloid cells that participate in the immunologic response to parasites and hypersensitivity reactions. ${ }^{1}$ Hypereosinophilia, defined as an absolute eosinophilic count of $1.5 \times 10^{9} / \mathrm{L}$ or greater, has a wide differential diagnosis (Box 1). ${ }^{2}$ Importantly, these cells can infiltrate tissues and cause end-organ damage, resulting in a rare condition known as hypereosinophilic syndrome, which has an estimated annual incidence of 0.035 per $100000 . .^{1,3,6}$ Although this multisystem condition most commonly involves the skin, gastrointestinal tract and the lungs, hypereosinophilic syndrome can result in cardiac manifestations in about $20 \%-50 \%$ of cases. ${ }^{1,6}$ Eosinophilic cardiac

Box 1: Causes of eosinophilia and prevalence in histologically proven eosinophilic myocarditis ${ }^{1-5}$

\section{Causes of eosinophilia}

\section{Idiopathic}

Hypersensitivity

Medications

- Antibiotics (e.g., penicillin and cephalosporin)

- Antiepileptic (e.g., phenytoin, lamotrigine and valproic acid)

- Anti-inflammatory (e.g., NSAIDs)

- Antihypertensives (e.g., hydrochlorothiazide, chlorthalidone and spironolactone)

- Sulfonamides

Allergies

- Chronic sinusitis (e.g., polypoid)

- Atopy (e.g., asthma and allergic rhinitis)

- Eosinophilic pulmonary disorders (e.g., eosinophilic pneumonia)

Eosinophilic gastrointestinal disorders (e.g., eosinophilic esophagitis)

\section{Rheumatologic and autoimmune}

- Antineutrophil cytoplasmic autoantibody-associated vasculitis (e.g., eosinophilic granulomatosis with polyangiitis and granulomatosis with polyangiitis)

- Systemic lupus erythematosus

- Severe rheumatoid arthritis

- Dermatomyositis

- Systemic sclerosis

- IgG4-related disease

- Inflammatory bowel disease

- Sarcoidosis

\section{Primary and neoplastic}

- Primary hypereosinophilic syndromes with molecular abnormalities (e.g., mutations of the PDGFRA, PDGFRB, FGFR1, PCM1 and JAK2 genes)

- Eosinophilic leukemia

- Lymphoma (e.g., B- and T-cell lymphoma, and Sézary syndrome)

- Solid tumours (e.g., gastrointestinal, lung and squamous epithelial cancers)

Infectious

- Parasites (e.g., Strongyloides, Schistosoma and Toxocara)

- Protozoan (e.g., Dientamoeba)

- Fungi (e.g., coccidioidomycosis and aspergillosis)

- Viral (e.g., HIV)

\section{Others}

- Adrenal insufficiency

- Immunodeficiency (e.g., autosomal-dominant hyper-IgE syndrome)

- Transplant rejection or graft-versus-host disease

- Cholesterol emboli

- Radiation

Note: IgG4 = immunoglobulin G4, IgE = immunoglobulin E, NSAID = nonsteroidal antiinflammatory drug.

$\%$ in eosinophilic myocarditis 
disease - also known as Loeffler endocarditis, eosinophilic myocarditis or fibroplastic endocarditis - is a major source of morbidity and mortality in hypereosinophilic syndrome. ${ }^{4,6}$

Most cases of eosinophilic myocarditis described in the literature have presented with symptoms of cardiac failure. ${ }^{6}$ Our case shows an alternative presentation, which is supported by the underlying pathophysiology. Historically, eosinophilic myocarditis is characterized by 3 phases (Box 2). ${ }^{7}$ The first phase of acute necrosis, typically asymptomatic, is caused by infiltration and subsequent degranulation of eosinophils in the myocardium. The ensuing damage to the ventricular wall results in the second stage of eosinophilic myocarditis, known as the thrombotic stage, which is characterized by the development of thrombi along the endocardium (Figure 1). Previous reviews have shown that ischemic complications, such as the multifocal strokes encountered in our case, occur during this phase in only $4 \%-12 \%$ of cases. ${ }^{4,7}$ Replacement of the thrombus with fibrosis signals the third and final stage of eosinophilic myocarditis. Most reported cases of eosinophilic myocarditis present in this phase with symptoms of heart failure secondary to restrictive cardiomyopathy: dyspnea, chest pain and cough. ${ }^{4,6,7}$ Interestingly, our patient had no signs of heart failure on presentation, and her initial echocardiogram was essentially normal. This highlights the need for clinicians to be aware of the heterogeneity in the presentation of this condition.

\section{Box 2: The 3 pathologic and clinical stages of eosinophilic myocarditis ${ }^{7}$}

\begin{tabular}{|c|c|}
\hline Stage & Pathologic and clinical manifestations of stage \\
\hline Acute necrosis & $\begin{array}{l}\text { - Infiltration of eosinophils to myocardium with subsequent degranulation of toxic proteins } \\
\text { - Usually asymptomatic, but myocardial necrosis can potentially be seen biochemically and } \\
\text { pathologically }\end{array}$ \\
\hline Thrombotic & $\begin{array}{l}\text { - Formation of mural thrombi along the damaged endocardium, most characteristic at the apex of } \\
\text { the ventricles } \\
\text { - Can result in secondary embolic phenomenon in about } 10 \% \text { of cases, including strokes and } \\
\text { myocardial infarctions }\end{array}$ \\
\hline Fibrotic & $\begin{array}{l}\text { - Damaged endocardium results in irreversible fibrosis and scarring, resulting in a restrictive } \\
\text { cardiomyopathy and restrictive valvular movements and regurgitation (e.g., mitral) } \\
\text { - Most commonly reported presentation of eosinophilic myocarditis with left- or right-sided heart } \\
\text { failure }\end{array}$ \\
\hline
\end{tabular}

Box 3: Recommended workup for hypereosinophilic syndrome ${ }^{2 *}$

\section{Category}

General biochemistry

Imaging and functional tests

Imaging and functional tests

Hematologic

Rheumatologic

Infectious

Biopsy
Investigations for hypereosinophilic syndrome

- Complete blood count, electrolytes and creatinine

- Liver enzymes plus bilirubin, lactate dehydrogenase and albumin

- Calcium

- Troponin

- C-reactive protein

- Echocardiography, cardiac magnetic resonance imaging or both

- Computed tomography of the chest, abdomen and pelvis (e.g., lymphadenopathy and neoplasms)

- Pulmonary function tests

- Serum and urine protein electrophoresis

- Quantitative immunoglobulins

- Peripheral blood smear

- Flow cytometry (blood and marrow)

- Molecular studies (e.g., PDGFRA, FGFR1 and JAK2) if eosinophilic count is greater than $1.5 \times 10^{9} / \mathrm{L}$

- Vitamin $B_{12}$ and tryptase levels (elevated in myeloid variants of hypereosinophilic syndrome)

- Antinuclear antibody and extractable nuclear antigens

- Antineutrophil cytoplasmic antibodies

- Rheumatoid factor and anti-cyclic citrullinated peptide

- Blood cultures

- Urinalysis and urine culture

- Stool culture, and examination for ova and parasites

- Serology for parasites or fungal infections (e.g., Strongyloides)

- HIV

- Biopsy of affected tissues

- Bone marrow biopsy (especially in the absence of identifiable causes)

*Investigations should be guided by history and physical examination for both causes and complications, in addition to a thorough review of allergic, drug and infectious etiologies. 
A high index of suspicion for cardiac involvement should be maintained when patients present with hypereosinophilia and signs of end-organ dysfunction or ischemic complications.

Cardiac MRI has emerged as a novel modality to help diagnose eosinophilic myocarditis because of its superiority in detecting inflammation, thrombi and fibrosis. Unfortunately, these imaging changes remain inconsistent in the early phases of eosinophilic myocarditis. ${ }^{1,6}$ More importantly, patients presenting with cognitive dysfunction may not be able to follow the commands needed to effectively conduct an MRI examination. Therefore, endomyocardial biopsy remains the gold standard diagnostic modality for eosinophilic myocarditis. ${ }^{7}$

A diagnosis of eosinophilic myocarditis should also prompt a comprehensive investigation for potential underlying primary and secondary etiologies (Box 1, Box 3). Primary hypereosinophilic syndrome typically occurs in the setting of an underlying hematologic malignancy, resulting in a monoclonal proliferation of eosinophils. These may then be further subclassified (per the World Health Organization) based on molecular abnormalities, such as mutations of the JAK2 or PDGFRA genes. ${ }^{3}$ Conversely, secondary etiologies - which result in polyclonal expansion of eosinophils - include autoimmune and rheumatologic disorders and exposures to parasites, allergens and medications. ${ }^{2}$ Numerous medications may cause eosinophilia, but they mostly include antibiotics, anticonvulsants, anti-inflammatories and antihypertensives. ${ }^{6,8}$ Although the latter include angiotensin-converting enzyme inhibitors, which our patient was taking (perindopril), most reported cases are associated with captopril and enalapril. ${ }^{8}$ Despite thorough investigation and removal of possible offending medications, most cases of eosinophilic myocarditis are idiopathic (as with our patient). 4,6

Management of eosinophilic myocarditis relies on removal of any precipitants (e.g., an allergic cause) and treatment of underlying causes, such as the use of tyrosine kinase inhibitors for clonal disorders (e.g., PDGFRA positive). Corticosteroids, at 0.5$1.0 \mathrm{mg} / \mathrm{kg}$ for about 2 weeks and tapered over 2-3 months to a maintenance dose of $5-10 \mathrm{mg} / \mathrm{d}$, are the cornerstone of treatment for idiopathic cases to decrease hypereosinophilia and the resulting end-organ damage, but evidence is limited to small case series. ${ }^{1,2,6}$ Second-line options include hydroxyurea and interferon- $\alpha .{ }^{1}$ Monitoring with echocardiography or cardiac MRI is indicated every 6 months in cases of sustained hypereosinophilia, and more frequently if cardiac disease is discovered. ${ }^{7}$ Thrombotic complications should be managed with anticoagulation in the form of low-molecular-weight heparin or warfarin, but there is little guidance behind the recommended duration and their prophylactic use..$^{4,6,7}$ Direct oral anticoagulants have not been studied for this indication. Congestive heart failure (from the fibrotic stage of eosinophilic myocarditis) should be treated based on current heart failure guidelines. Valvular involvement may require surgical interventions. ${ }^{4,7}$ Long-term outcomes in these patients remain unknown.

\section{References}

1. Cheung CC, Constantine M, Ahmadi A, et al. Eosinophilic myocarditis. Am J Med Sci 2017;354:486-92.

2. Butt NM, Lambert J, Ali S, et al. Guideline for the investigation and management of eosinophilia. Br J Haematol 2017;176:553-72.

3. Gotlib J. World Health Organization - defined eosinophilic disorders: 2017 update on diagnosis, risk stratification, and management. Am J Hematol 2017;92:1243-59.

4. Brambatti M, Matassini MV, Adler ED, et al. Eosinophilic myocarditis: characteristics, treatment, and outcomes. J Am Coll Cardiol 2017;70:2363-75.

5. Kovalszki A, Weller PF. Eosinophilia. Prim Care 2016;43:607-17.

6. Mankad R, Bonnichsen C, Mankad S. Hypereosinophilic syndrome: cardiac diagnosis and management. Heart 2016;102:100-6.

7. Ogbogu PU, Rosing DR, Horne MK. Cardiovascular manifestations of hypereosinophilic syndromes. Immunol Allergy Clin North Am 2007;27:457-75.

8. Rauscher C, Freeman A. Drug-induced eosinophilia. Allergy Asthma Proc 2018;39:252-6.

\section{Competing interests: None declared.}

This article has been peer reviewed.

The authors have obtained patient consent.

Affiliations: Department of Medicine (Chan), University of British Columbia, Vancouver, BC; Department of Medicine (Gibson), Cumming School of Medicine, University of Calgary, Calgary, Alta.

Contributors: Ka Hong Chan drafted the manuscript, which Paul Gibson reviewed. Both authors gave final approval of the version to be published and agreed to be accountable for all aspects of the work.

Acknowledgement: The authors thank Dr. Yinong Wang for his support in obtaining the pictures of the biopsy.

Correspondence to: Paul Gibson, gibsonp@ucalgary.ca

The section Cases presents brief case reports that convey clear, practical lessons. Preference is given to common presentations of important rare conditions, and important unusual presentations of common problems. Articles start with a case presentation (500 words maximum), and a discussion of the underlying condition follows (1000 words maximum). Visual elements (e.g., tables of the differential diagnosis, clinical features or diagnostic approach) are encouraged. Consent from patients for publication of their story is a necessity. See information for authors at www.cmaj.ca. 